\section{Border defense}

Some plants are resistant to bacteria painted on the leaf surface but are susceptible to the same bacteria injected directly into the leaf. In Cell, He and colleagues find that bacteria use plant stomata, which routinely open and close in response to fluctuations in light and humidity, as portals of entry into the leaf intercellular space. After detecting flagellin or lipopolysaccharide, arabidopsis guard cells close stomata. Bacteria-induced sealing of stomata requires components of the abscisic acid signaling pathway, which also regulates the responsiveness of stomata to humidity. However, bacteria expressing the established phytotoxin COR ultimately inhibit abscisic acid signaling and trigger stomata reopening. COR-mutant bacteria cause disease in arabidopsis strains with mutations in genes encoding stomata closure machinery, but are avirulent when spread on the surface of wild-type arabidopsis. These data suggest that pathogens devised strategies to subvert stomata defenses during the evolutionary 'tug-of-war' between plants and pathogens.

$C B$ Cell 126, 969-980 (2006)

\section{Activating caspase-1}

Caspase- 1 is needed to convert inactive interleukin $1 \beta$ (IL-1 $\beta$ ) and IL-18 precursors into potent proinflammatory mediators that are secreted into surrounding tissues. Cytosolic duplex RNA resembling double-stranded RNA (dsRNA) viruses can trigger caspase-1 activation, but the signaling pathway remains to be elucidated. In the Journal of Biological Chemistry, Kanneganti et al. show that caspase-1 activation by dsRNA requires the cytosolic Nod-like family member cryopyrin (also called NALP3). Notably, caspase- 1 activation is unimpaired in macrophages deficient in other dsRNA sensors such as Nod 2 or Toll-like receptor 3 or 7; conversely, cryopyrin-deficient mice do not alter their elaboration of tumor necrosis factor in response to poly(I:C). These data suggest the 'inflammasome' complex, containing cryopyrin and the adaptor ASC, is specifically required for caspase-1-mediated cleavage of IL-1 $\beta$ and IL-18 proforms in response to dsRNA.

J. Biol. Chem. (28 September 2006) doi:10.1074/jbc.M607594200

\section{Evading Nods}

Heliobacter pylori bacteria, which colonize the human stomach, can convert between spiral and coccoid forms in mice. The latter form is associated with the accumulation of a GM-dipeptide motif in the peptidoglycan layer. In PLoS Pathogens, Boneca and colleagues examine the molecular mechanisms involved in that conversion. Unlike wild-type counterparts, $\boldsymbol{H}$. pylori containing a mutation in the gene encoding AmiA, an established $\mathrm{N}$-acetylmuramoyl-Lalanine amidase, fail to accumulate the GM-dipeptide motif and do not convert to the coccoid form during the stationary phase in vitro. Transition to the coccoid form is accompanied by loss of the GM-tripeptide motif, which is recognized by Nod1 expressed in gastric epithelial cells. Notably, Nod2 recognizes muropeptides containing the GM-dipeptide motif but not naturally occurring anyhdromuropeptide byproducts thereof. These data suggest that adoption of the coccoid form may allow $\boldsymbol{H}$. pylori to evade detection by Nod 1 and Nod2 and emphasize the crucial immune defense function of Nod proteins. PLoS Pathog. 2, 844-852 (2006)

Research notes written by Christine Borowski, Douglas C. Braaten and Laurie A. Dempsey

\section{Another pattern receptor}

Invasive pneumococcal infection requires host expression of platelet-activating factor receptor (PAFr), a G protein-coupled receptor that recognizes phosphorylcholine motifs. In Journal of Immunology, Fillon et al. find that PAFr is required for the severe pathophysiology associated with the accumulation of bacterial wall 'debris', generated during antibiotic therapy, for example, in cells and on epithelial surfaces. Bacterial cell wall components bind PAFr expressed on host cells; the accumulation of debris causes inflammation and tissue damage in some organs. Mice infected with pneumococcus have lethal cell wall accumulations on vascular endothelium that require expression of PAFr but not Toll-like receptor 2 or Nod2. Cell wall accumulations are found in both brain and heart cells; these lead to $\beta$-arrestin expression and little inflammation in the former, and phospholipase $\mathrm{C}$ signaling and loss of contractility in the latter. Cardiocyte damage is ameliorated if an antagonist of PAFr is introduced before cell wall challenge. PAFr thus functions as innate pattern-recognition receptor to induce inflammation.

J. Immunol. 177, 6182-6191 (2006)

\section{RIG-I binds 5' triphosphates}

The RNA helicase RIG-I senses the presence of certain viral genomes after cytoplasmic infection and activates a potent antiviral response. However, the actual ligand recognized by RIG-I has remained unknown. In Science, Hornung et al. and Pichlmair et al. identify RNA bearing unmodified 5 ' triphosphate residues, which differs from typical eukaryotic 7-methyl-guanosine 'capped' mRNA, as the ligand recognized by RIG-I. Monocytes transfected with RNA constructs with $5^{\prime}$ triphosphate ends elicit abundant interferon- $\alpha$ production dependent on the expression of RIG-I but not that of the related intracellular sensor Mda5. RIG-I is also needed for the production of interferon- $\alpha$ after infection by rabies virus, which has a genome with a $5^{\prime}$ triphosphate end, but not picornaviruses, which bear protein-conjugated 5' ends. RIG-I might have arisen to specifically recognize cytosolic RNA structures that differ substantially from those produced by nuclear transcription and subsequent modification in host cells.

Science (12 October 2006) doi:10.1126/science1132505 \& doi: $10.1126 /$ science. 1132998

\section{Tomato immunity}

Infection of tomatoes by Pseudomonas syringae pathovar tomato triggers disease resistance mediated by the plant protein kinase Pto and the plant nucleotide-binding site-leucine-rich repeat (NBS-LRR) protein Prf. In The Plant Cell, Rathjen and colleagues evaluate the relationship between Pto kinase and Prf during both pathogen-dependent and pathogen-independent signaling. In the former, pseudomonas effector proteins interact directly with Pto, expression of Prf is required for normal resistance function and both proteins contribute to effector recognition. In the absence of infection, overexpression of either Pto or Prf in the plant Nicotiana benthamiana causes Prf activation. Pto and Prf physically interact and are found in high-molecular-weight 'recognition' complexes. Although constitutively active Pto expressed in the absence of Prf does not signal, highly overexpressed Pfr alone does, indicating that Pfr is the signal transducer of the Pto-Prf duplex. The essential interplay between host NBS-LRR and host kinase described may be paradigmatic of 'indirect' signaling mediated by other Nod-like proteins, such as those in mammals. $D C B$ Plant Cell (6 October 2006) doi:10.1105/tpc.106.044016 